\title{
Leonhard Euler's "principle of mechanics" (an essay on the foundations of the equations of motion)
}

\author{
Penha Maria Cardozo Dias* \\ Universidade Federal do Rio de Janeiro, Rio de Janeiro, RJ, Brasil
}

Received on February 15, 2017. Accepted on April 04, 2017.

\begin{abstract}
Leonhard Euler derived equations of motion for both (in modern terminology) point mass mechanics and analytic mechanics. In order to derive the equations, some dynamic premise has to be introduced; this is the "principle of mechanics". It stems from the recognition that infinitesimal motions are uniformly accelerated. Then, using Galileo Galilei's theorem on the fall, mathematical relations among differentials acquire physical meaning, and become the "principle of mechanics".
\end{abstract}

Keywords: physical meaning of the lagrangian, differential equations of motion, Leonhard Euler

\section{Introduction}

This paper is broadly speaking a search for the arguments on which the categories of dynamic are founded; for instance: why is mechanics written in the language of mathematics? why are the laws expressed by differential equations? why are these second order in time? what is the physical meaning of the energy $T-V$ (the meaning must be very important, because from it the equations are obtained)? The list is huge. The history of physics provides elements for an analysis of the foundations of physics: it discloses arguments (whether logical or empirical), questions for whose solution some category is proposed, clarify concepts, showing the "whys and wherefores" and the "hows". This is better done in case studies, by analyzing arguments rather than pertinence to general contexts 1 tackling foundations questions "at large" risks to conflate approaches that are conceptually distinct, and to neglect mathematical calculations that not only clarify, but in many cases give meaning to ideas. In this paper, a case study is used to investigate the foundations of the laws of motion. The case study is Leonhard Euler's "principle of mechanics": in the absence of

*Endereço de correspondência: penha@if.ufrj.br

${ }^{1}$ Many (if not all) questions about the foundations of physics require conceptual analysis, i.e., discussion of the necessity of a concept in a given context of categories, of the meaning of concepts, of the way a problem is stated and its relation to available mathematical tools, etc. But there is no conceptual analysis without an archive of facts, which sets a context that defines the background or arena where scientists and science dwell. However, the fashion among historians is the historiography of science: relations among scientists — as expressed in citations, similarity of methods and research, and their social interaction — are searched for, therefrom writing a description "at large" of the flux and transformation of ideas; as necessary as it may be, the mere description of facts cannot account for those specific arguments that make an idea appealing to a particular individual, and for it being fruitful. a general dynamic, some premise has to play a dynamic role; the paper is a search for the dynamic premise in the "principle of mechanics".

In Recherches and in Découverte [1,2], Euler derives three differential equations (one for each coordinate) to describe the motion of a (modern terminology) point mass; in modern notation, this is the equation $\vec{F}=m \frac{d^{2} \vec{r}}{d t^{2}}$. In Réflexions and in Harmonie [3 4], Euler derives equations for (modern terminology) analytical mechanics. In all these papers, the equations follow from the same dynamic premise; in Harmonie [4] Euler calls it the "principle of mechanics". This principle is first deduced in the earlier Mechanica [5], starting from properties of differentials; in later papers, Euler inverts the reasoning, deducing equations of motion from the "principle of mechanics". It is claimed:

1. Properties of some differentials disclose the "principle of mechanics" (sections 2, 3 and 4): it is the law of the uniformly accelerated motion stated by Galileo 6 9 $92^{2}$ as Euler recognizes in $\$ 135$ in the Mechanica.

2. The mathematical relation that issues from the properties of differentials becomes the "principle

\footnotetext{
${ }^{2}$ In the Two New Sciences 6], Galileo deduces expressions for an uniformly accelerated, straight line motion. He invokes the medieval "theorem of the mean speed" to prove that (in modern notation) $s \propto t^{2}$, and as a corollary that $s \propto v^{2}$, where $s$ is the distance moved in time $t$, and $v$ is the speed at $t$ (if the body is released from rest). The medieval theorem states that the distance moved in an uniformly accelerated motion is equal to the distance moved in an uniform motion with a speed equal to the mean speed in the accelerated motion; it was formulated in the fourteenth century, in a different context, the problem of the increase and remission of qualities (7], p.199-241; 8], p.185-200). However the application of the medieval theorem to find mathematical expressions for the fall on the surface of the Earth was done two hundred years later by Galileo (9], p.203).
} 
of mechanics", only when the terms that enter the relation are interpreted according to the terms in Galileo's theorem (section 5).

3. In section 6 , the import of the "principle of mechanics" to the foundations of physics is discussed. Any 'force', whatever its origin, not only is measured by a weight, but has the nature of a weight ${ }^{3}$

By the time Euler wrote his Mechanica, other authors had already produced the differential form of Isaac Newton's axiom II: in his 1716 Phoronomia, Jacob Hermann stated a lemma to the effect that (his notation) " $G=M V: T$ ", then he generalized it into a differential expression; Joseph-Louis Lagrange considered that Colin MacLaurin was "... the first to employ [the equations] in his Traité des Fluxions" [10]4 Euler himself proposes the analytical method, after criticizing Isaac Newton's "old synthetic [geometric] method" $(\sqrt{5}, \mathrm{p} .8)$ :

\section{$[\ldots]$ as with all writings composed without analysis, and that mainly falls to be the lot of Mechanics, $[\ldots]$, an examination of these propositions cannot be followed with sufficient clarity and distinction $[\ldots]$. Thus, I always have the same trouble, when I might chance to glance through Newton's Principia or Hermann's Phoronomia, [...] that whenever the solutions of problems seem to be sufficiently well understood by me, that yet by making only a small change, I might not be able to solve the new problem using this method. Thus I have endeavored [f] or a long time now, to use the old synthetic method to elicit the same propositions that are more readily handled by my own analytical method, and so by working with this latter method I have gained a perceptible increase in my understanding.}

Years later, in 1747, Euler proposes to apply to the motion of the planets ([1, p.100) "some new discoveries in Analysis"; then he deduces from the "principle of mechanics" the "general and fundamental principle of the entire mechanics", as he calls the equation.

\footnotetext{
${ }^{3}$ This is curious, because the legend in physics departments is that the "inertial mass" and the "gravitational mass" are united only in the early twentieth-century, by the "principle of equivalence". My guess is that instrumentalism must have had an influence in the supposition of two different kind of masses.

${ }^{4}$ However, to what use was the differential form of the axiom put? According to Bernard Cohen ([1], p.158), axiom II is better understood as a definition. Writing axiom II in the language of the leibnizian calculus is not enough to change its status from that of a definition into that of an equation of motion. MacLaurin states the differential form very late in his book ( $\$ 884$, volume 2, p.298). Clifford A. Truesdell (12], p.251) claims that the publication in 1746 of the partial differential equation of the string (wave equation) by Jean Le-Rond D'Alembert, and Johann Bernoulli's Hydraulica (1739) "must have had a sensational effect" on Euler; the "effect" was the Recherches.
}

\section{The "principle of mechanics" in the "Réflexions" and in the "Harmonie"}

In 1740, Pierre-Louis-Moreau de Maupertuis founded statics on the "principle of rest", and in 1744 he founded dynamics on the "principle of least action". In Réflexions and in Harmonie 3, 4, Euler put the two principles in "harmony", from which the principles of analytical mechanics followed. Simplifying a lengthy discussion Euler states "harmony" as $13: 5$

$$
(\text { action })=C t-(\text { effort })
$$

where $C$ is a constant, and:

$$
\begin{aligned}
\text { action }= & +\int d t T \text { where } \delta T=M \vec{v} . \delta \vec{v} \\
& \text { and } T=\frac{1}{2} M v^{2} \\
\text { effort }= & -\int d t V \quad \text { where } \delta V=-\vec{F} \cdot \delta \vec{s} \\
& \text { and } V=-\int \vec{F} \cdot \delta \vec{s}=- \text { work }
\end{aligned}
$$

Eq. (1) follows from the "principle of mechanics" 4$]$ which is (p.156, already correcting factors and modernizing the notation):

$$
M \vec{v} . \delta \vec{v}=-\vec{F} . \delta \vec{s} ; \quad \text { or } \quad \delta T=\delta V .
$$

Euler calls $\delta V=-\vec{F} . \delta \vec{s}$ "the height due to the speed". Then the "principle of mechanics", Eq. (2), means 13 the motion on an infinitesimal segment $(\delta s)$ of the trajectory is an uniformly accelerated "fall", so that after a time interval $d t$, the "available height" $(\delta V)$ is entirely converted into "motion" $(\delta T)$. The principle holds in $d t$ only, so that all the $d t$ s are taken into account by an integration: $\int d t \delta(T-V) \equiv \delta \int d t(T-V)=0$.

\section{The "principle of mechanics" in the "Recherches" and in the "Découverte"}

Euler introduces the three coordinates representation of the differential equation $\vec{F}=m \frac{d^{2} \vec{r}}{d t^{2}}$ in $\S 18$ of Recherches, and again in Découverte [1,2]. He proposes to find the solution to the following problem $([1]$, p.102):

\footnotetext{
18. LEMMA. If a body at $M$ is acted on by any forces, find the instantaneous change on the motion of the body produced by these forces.
}

\footnotetext{
5 "Harmony" is found in physics books 14. Calling $\vec{F}_{A}$ the applied force on the particle $A$, and the constraint force $\vec{f}_{A}$, then on the principle that the work done by the constraint forces is zero, the total work done on or by the system of $N$ point masses is: $\sum_{A}\left(\vec{F}_{A}-m_{A} \frac{d \dot{\vec{r}}_{A}}{d t}\right) \cdot \delta \vec{r}_{A}=\sum_{A} \vec{f}_{A} \cdot \delta \vec{r}_{A} \equiv 0$. Transforming cartesian coordinates into configuration and momentum coordinates, one obtains: $\delta T=+\delta V+\frac{d}{d t} \sum_{A}\left(p_{A} \delta q_{A}\right)$, which is (1) after integration in $t$ and $\delta$; also $C=\sum_{A} \int_{\left(q_{A}\right)_{\text {finitiol }}}^{\left(q_{A}\right)_{\text {final }}}\left(p_{A} \delta q_{A}\right)$. If $(\delta q)_{\text {initial }}=(\delta q)_{\text {final }}=0$, as done in the $\delta$-variation, then $C=0$.
} 
The solution is $([1], \mathrm{p} .103)$ :

SOLUTION. [...] taking the element of time $d t$ as constant, the instantaneous change of motion of the body is expressed by the three equations:

I. $2 \frac{d d x}{d t^{2}}=\frac{X}{M} ; \quad$ II. $2 \frac{d d y}{d t^{2}}=\frac{Y}{M} ; \quad$ III. $2 \frac{d d z}{d t^{2}}=\frac{Z}{M}$.

It is possible to find from them the values $x, y, z$, for any elapsed time $t$, consequently the place where the body is.

where: $X, Y$ and $Z$ are the components of the "absolute or motive force" on the three coordinates axes; $M$ is the mass.

The solution demands justification. It is significant that instead of the correct equation $\left(\frac{d^{2} \vec{r}}{d t^{2}}=\frac{\vec{F}}{M}\right)$, Euler proposes (in modern notation) $2 \times \frac{d^{2} \vec{r}}{d t^{2}}=\frac{\vec{F}}{M}$. The factor 2 in front of the equation hints to the justification of the equation ${ }^{6}$ in fact, the corollary that follows $\$ 18$ leaves no doubt as to the origin of the factor, and to the interpretation of the paragraph $([1]$, p.103):

19. COROLLARY 1. The speed of the body along the direction $[x]$ is $\frac{d x}{d t}$, along $[y], \frac{d y}{d t}$ and along $[z]$, it is $\frac{d z}{d t}$; then the squares of these formula, that is, $\left(\frac{d x}{d t}\right)^{2},\left(\frac{d y}{d t}\right)^{2}$ and $\left(\frac{d z}{d t}\right)^{2}$ are the heights corresponding to these speeds. Because of these relations, the [derivatives] are multiplied by the number 2 .

The corollary makes a unambiguous reference to an uniformly accelerated motion, in the form $v^{2} \propto x$ or $v^{2}=\mathcal{G} x$, that is to say, to the "principle of mechanics"; it also indicates the following calculation:

principle of mechanics: $\quad\left(\frac{d x}{d t}\right)^{2}=\mathcal{G} x$

first derivative: $2\left(\frac{d x}{d t}\right)\left(\frac{d^{2} x}{d t^{2}}\right)=\mathcal{G}\left(\frac{d x}{d t}\right)$

cancelling terms: $\quad 2\left(\frac{d^{2} x}{d t^{2}}\right)=\mathcal{G}$

Then Euler makes $\mathcal{G}=\frac{\text { force }}{\text { mass }}$. This needs justification; instead, Euler includes a paragraph to the effect that $\mathcal{G}$ has units of acceleration $\left([1]\right.$, p.104) ${ }^{7}$

${ }^{6}$ The factor 2 is cumbersome: sometimes it correctly divides $v^{2}$ (as in Réflexions, p.55), in other occasions it multiplies $v^{2}$ (as in Recherches and in Découverte), or it is simply absent (as in Harmonie, p.158).

${ }^{7}$ In Mechanica, Euler uses $p$ for "force", but the $p$ in $\S 22$ is better understood as acceleration: $u$ is speed, $s$ is distance, and in $\S 18$, when Euler refers to the force, he uses the words "absolute or motive", not "accelerative power", as he calls $p$ in $\$ 22$. However, it may be argued that Euler is invoking the expression for accelerated motions on straight lines $\frac{d v}{d t}=n\left(\frac{F}{M}\right)$, or $\frac{d^{2} s}{d t^{2}}=n\left(\frac{F}{M}\right)$, formerly derived in the Mechanica, in which case it can be considered known that acceleration is measured in units of $n \frac{F}{M}$. But in the second half of the quote, Euler warns that "it is necessary some thought to realize that this principle extends equally to every partial motion
22. SCHOLIUM. This lemma is founded on nothing else than the principle known in mechanics, $d u=p d t$, where $p$ denotes the accelerative power and $u$ the speed; because, if $u=\frac{d s}{d t}$, and if $d s$ is the element of the distance [moved by the body], then it is $\frac{d u}{d t}=\frac{d^{2} s}{d t^{2}}$ and then $\frac{d^{2} s}{d t^{2}}=p$. But it is necessary some thought to realize that this principle extends equally to every partial motion into which the real motion [can be] reduced in thought; otherwise this lemma contains at the same time all the principles that are commonly used to find curved motions.

Euler stills owes an argument to the effect that acceleration can be measured in units of $\frac{\text { force }}{\text { mass }}$.

The argument first stated in the Mechanica (section $5)$ is repeated in the later Découverte ([2], p.89):

21. In order to understand the strength of this formula $\left[\mathrm{M} d^{2} x= \pm \mathrm{P} d t^{2}\right]$, it is necessary to explain to what units the various quantities $M, P, x$ and $t$, refer. To start with, it has to be noted that insofar $M$ denotes the mass of the body, it also expresses at the same time the weight of the body on the surface of the Earth; the force $P$ being reduced to the weight, the letters $M$ and $P$ contain homogeneous quantities. Then: the speed of the body in its motion [...] is $\frac{d x}{d t}$, and if we suppose that this speed is equal [to the speed] a heavy body acquires when it falls from the height $[x]$, it is necessary to take $\left[\left(\frac{d x}{d t}\right)^{2}=x\right]$ or $\left[d t=\frac{d x}{\sqrt{x}}\right]$; from the latter, the relation between the time $t$ and the distance $x$ is known [after integration].

That is, in an infinitesimal time interval the force is constant, and can be measured by the weight, $W=M g$, of the moving body; then there must exist a "local and instantaneous constant" $\lambda \equiv \lambda(\vec{r}, t)$ such that at each point on the trajectory:

$$
\begin{aligned}
\text { measure of } F & =\lambda(\vec{r}, t) \times W \\
\text { measure of } M & =\frac{W}{g} \\
\text { measure of } \frac{F}{M} & =\lambda(\vec{r}, t) \times g \equiv \mathcal{G}(\vec{r}, t) .
\end{aligned}
$$

The above derivation of the law can be interpreted: the accelerated infinitesimal motion along a coordinate axis can be considered as a "fall" due to the "weight" $F=\lambda W$, with acceleration due to the "gravity" $\mathcal{G}=$ $\lambda g$. Anticipating results in section 5, the "principle of mechanics" is best understood as:

$$
d\left(v^{2}(\vec{r}, t)\right)=2 g \lambda(\vec{r}, t) d s
$$

into which the real motion [can be] reduced in thought"; that is to say, the purpose of $\S 18$ is to deduce an equation true for any accelerated motion on any curve, not only for the infinitesimal straight line motion (to which $v^{2}=\mathcal{G} x$ applies) "into which the real motion [can be] reduced in thought". The mentioned "necessary thought" is developed in the earlier Mechanica, but is not presented in the Recherches. 
where $d s$ is the infinitesimal distance moved on the trajectory; making $\mathcal{G} \equiv g \lambda(\vec{r}, t)$, the "principle of mechanics" is $d\left(v^{2}\right)=2 \mathcal{G} d \nu$, where $d \nu \equiv d s$ is measured in dimension of "height".

It should be mentioned that on the historical point of view, $W=M g$ is an assumption weaker than $F=M \mathcal{G}$ : in fact, the gravitational fall had been known to be accelerated since old 7. 8]; furthermore that the acceleration is constant and that it independs of the "body" is contained in Galileo's theorem, which is recognized by Euler in the Mechanica $([5]$, p.52):

141. [...] [F]rom the weight of each body it is usual to investigate the mass, and it is agreed that the weight and the quantity of matter are in proportion. Moreover it is agreed by experiment that all bodies in an empty space fall equally, and therefore all are accelerated equally by the force of gravity.

\section{The "principle of mechanics" in the "Mechanica"}

In the Mechanica, Euler derives the law for accelerated motions on straight lines, and then uses it to obtain the law for accelerated motions on any line.

He starts by considering that "as in geometry the elements of curved lines are considered to be the elements of small straight lines" [5], so that motions on curved lines are formed by a succession of infinitesimal straight line motions; on each of these, the speed is uniform, i.e., $d s=($ constant $) \times d t \equiv v d t(\sqrt{5]}$, p. $20 ;$ p.21 $):$

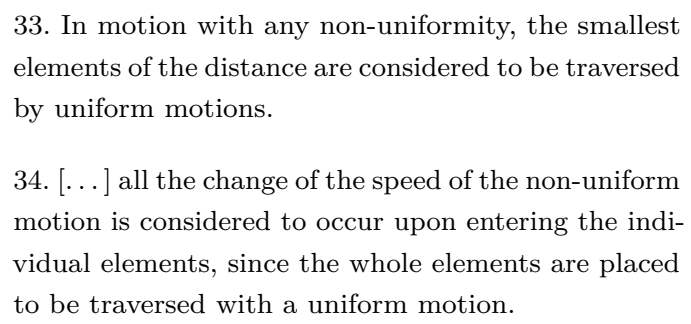

34. [...] all the change of the speed of the non-uniform motion is considered to occur upon entering the individual elements, since the whole elements are placed to be traversed with a uniform motion.

In the modern way of saying it, in first order approximation, if $j$ is the order of the segment: $s_{j+1}=s_{j}+v_{j} d t_{j}$ and $v_{j+1}=v_{j}+F_{j} d t_{j}$, where the force $F_{j}$ is constant in $d t_{j}$ (and acts all at once at the start of $d t$ ).

Second order differentials are then introduced (§130). If the motion were uniform, the mass would move $v d t$ in $d t$; due to the acceleration, it moves an extra $\delta$. According to $\S 34, \delta=d v d t$, as in the uniform motion; clearly, $\delta \equiv$ $d^{2} s$. While the infinitesimal motion is always uniformly accelerated, it need not be so in the finite motion $(\sqrt{5}]$, p.50):

134. [...] that the increments of the speed are in proportion with the increments of time in which they are generated $[d v=$ constant $\times d t]$ is also in agreement with finite quantities, as long as the force acting on the point stays the same, and always retains the same direction as the motion of the point itself.
Euler then shows that $d v \propto\left(\frac{F}{M}\right) d t$ : (i) it is proved that $F \propto M{ }^{8}$ (ii) it is proved that $\delta \propto F$ (Appendix A) ${ }^{9}$ (iii) then $F \propto M \delta$ or $F \propto M d v$ (because in a fixed interval $d t, \delta \propto d v)$ or $d v \propto \frac{F}{M}$; (iv) also $d v \propto d t(\S 134)$, so that $d v \propto \frac{F}{M} d t$. Finally, this is transformed into an equality by introducing a constant $n(\sqrt{5}$, p.57):

159. Since $\left[d v=n\left(\frac{F}{M}\right) d t\right]$, the distance through which the point $[M]$ is lead by the force $[F]$ in the element of time $d t$ will be equal to $\left[n\left(\frac{F}{M}\right) d t^{2}\right]$. This distance is indeed the product of $[d v]$ by $d t$. For by saying that this distance is $[\delta]$, then $\left[d v=\frac{\delta}{d t}\right](\S 128)$ and thus $\left[\delta=d v d t=n\left(\frac{F}{M}\right) d t^{2}\right]$.

Or in any straight line motion:

$$
d v=n\left(\frac{F}{M}\right) d t \quad \text { and } \quad v=n \int\left(\frac{F}{M}\right) d t
$$

equivalently, making $d t=\frac{d s}{v}$ :

$$
v d v=n\left(\frac{F}{M}\right) d s
$$

Euler is now ready to deduce the general differential relation in accelerated motions on any line. An arbitrary infinitesimal motion is illustrated in Fig.1.

The acceleration is along $\overline{A C}$, so that, from Eq. (3), left, (making $d v=\frac{\overline{A C}}{d t}$ ), the straight line infinitesimal distance moved in the direction of the force is given by $\overline{A C}=n\left(\frac{F}{M}\right) d t^{2}$. This result is used to prove that in first order (Appendix B):

$$
d v=n\left(\frac{F}{M}\right) d t \cos (\widehat{B D b})
$$

Then the general law of motion is stated $([5]$, p.58):

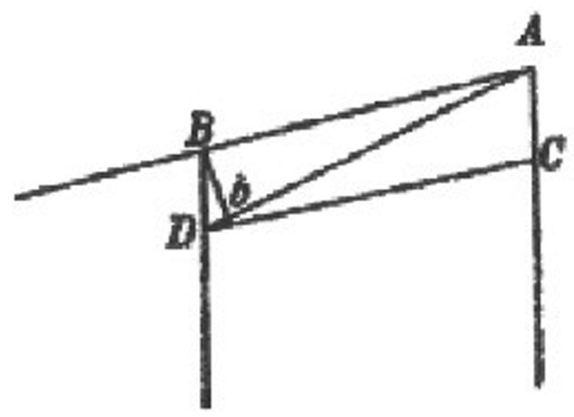

Figura 1: "The effect of an oblique force". $\overline{A B}=v d t$ is the distance moved with an uniform motion along the tangent to the curve. $\overline{A C}$ is the distance moved due to a force acting along $A C$. The resulting motion is $\overline{A D}$. $B b \perp A D$.

\footnotetext{
${ }^{8}$ Paraphrasing $\S 136(\sqrt[5]{ }$, p. .57$)$, if the force $F$ acts on $M$, then the force $\frac{1}{M} \times F=\frac{F}{M}$ acts on each of the parts of $M$, "for they will always remain united if they were indeed connected together initially" (5], p.51)

${ }^{9}$ The proof does not involve any law of physics, which reinforces the point that so far the relations are entirely mathematical.
} 
161. The distance $A D$ is called $d s$ [Fig.1], and the element of time $[\ldots] d t=\frac{d s}{v}$, then with $\left[\frac{d s}{v}\right]$ in place of $d t$ above there is produced $\left[d v=n\left(\frac{F}{M}\right) \frac{d s}{v} \sqrt{1-k^{2}}\right]$. The perpendicular $D F$ is drawn from $D$ to the direction of the force $A E$; let $A F=d y$ and $D F=d x$, then $[\ldots] d s^{2}=d x^{2}+d y^{2}$ and $k=\frac{d x}{d s}$ and $\left[\sqrt{1-k^{2}}=\frac{d y}{d s}\right]$. Hence $[\ldots]\left[d v=n\left(\frac{F}{M}\right) \frac{d y}{v}\right]$ or $[M v d v=n F d y]$.

Or:

$$
v d v=n\left(\frac{F}{M}\right) d y \quad \text { or } \quad d v^{2}=2 n\left(\frac{F}{M}\right) d y
$$

\section{The fall on the Earth and the "principle of mechanics"}

The general law, Eq. (6), is deduced from mathematical relations among differentials; it is best taken as a kinematic relation, since no physical dynamic principle has been invoked, except for the existence of some $F$, which so far is just a name for the cause of acceleration. In this section, it is argued that Eq. (6) becomes a general dynamic principle, when its terms are interpreted according to Galileo's theorem; the nature of $F$ and $n$, and of their associations with physical entities are obtained by comparison with Galileo's theorem.

The basis of the interpretation of Eq. (6) is found in $\S 134$ (section 4 above): infinitesimal motions, whatever the force, are uniformly accelerated. So is the finite motion of a falling weight $([5]$, p.50):

135. Galileo was the first person to use [the proportionality of the speed and the time, $d v \propto d t]$ in the investigation of a falling weight, for the solution of the problem [...].

Then the strategy is to use the solution in the case of the gravitational fall to find $n$.

Accelerated motions on straight lines are treated in chapter 3 in the Mechanica. The solution of Eq. (3) for a constant $F$ is $([5]$, p. 67$)$ :

$$
v^{2}=2 n\left(\frac{F}{M}\right) x
$$

if the initial conditions are $x=0$ and $v=0$. This solution also holds for the gravitational fall $([\sqrt[5]]{3}, \mathrm{p} .68)$ :

196. This case agrees above all with the fall of bodies on the $[E]$ arth: for the force of gravity, which in turn takes the place of the force, is uniform for not too great distances from the surface of the earth. Therefore in the free fall of weights the speeds are as the square roots of the distances traversed. This is the proposal of Galileo himself, that he discovered first from experiment and then by reasoning. [...].

The constant acceleration of the fall on the surface of the Earth is $g=\frac{W}{M}([5]$, p.68):
197. In an empty space, which can be effected with the help of pneumatic pumps, it has been shown by many experiments that any bodies fall equally. From which it follows, if there should be no air, all bodies that fall from equal heights gain equal speeds. On account of this, if $[W]$ designates the force of gravity, by which any body $[M]$ is moved, then $\left[\frac{W}{M}\right]$ is always the same constant. Hence the force of gravity is proportional to the quantity of matter in the body on which it acts. But that force is none other than the weight of the body; [...].

Then in the case of the gravitational fall, Eq. (7) can be written:

$$
v^{2}=2 n\left(\frac{W}{M}\right) x=2 n g x ;
$$

making $n=\frac{1}{2 g}$, this expression becomes $v^{2}=x{ }^{10}$ Then in a gravitational fall $([5], \S 199$, p.68) "[...] it will be possible for the height to denote the square of the speed". Likewise, the "height due to (or corresponding to) the speed" is $([5]$, p.69):

200. Hereafter we will call the height corresponding to the speed that height, from which a weight falling to the surface of the [E]arth, acquires that same speed.

201. This height must therefore be as the square of the speed, to which it refers. With the speed $[v]$ arising and with the due height $[\nu],[\nu]$ shall be as $\left[v^{2}\right]$.

Having found that $n=\frac{1}{2 g}$, the general equation for straight line motions, whatever the force, Eq. (4), becomes

$$
v d v=\frac{1}{2 g}\left(\frac{F}{M}\right) d y \quad \text { or } \quad d\left(v^{2}\right)=\frac{1}{g}\left(\frac{F}{M}\right) d y
$$

making $g=\frac{W}{M}$ :

$$
d\left(v^{2}\right)=\left(\frac{F}{W}\right) d y
$$

analogy with the relation $v^{2}=x$ that holds in the gravitational fall implies that $d \nu=\left(\frac{F}{W}\right) d y$ must be a true height, so that $F$ has dimensions of $W$, i.e., $F=\lambda W$, where $\lambda$ is a constant.

Finally, the result is generalized to the case of a curved trajectory, Eq. (6) ([5], p.70):

208. In a like manner, indeed the equations set out in $(161[M v d v=n F d y])$ and (163 [the centripetal force]) $[\ldots]$, substituting $[\nu]$ in place of $\left[v^{2}\right]$ and $\left[\frac{1}{2 g}\right]$ in place of $n$, are transformed into $[g M d \nu=F d y]$ $[\ldots]$.

Making $n=\frac{1}{2 g}=\frac{1}{2} \frac{M}{W}$, the general law, Eq. (6), is:

$$
d v^{2}=\left(\frac{F}{g M}\right) d y=\left(\frac{F}{W}\right) d y
$$

\footnotetext{
${ }^{10} \overline{\text { Actually, Euler makes } \frac{W}{M}}=1$ or $g=1$, so that $n=\frac{1}{2}$.
} 
Again, because the infinitesimal motion is uniformly accelerated, $d \nu=\left(\frac{F}{W}\right) d y$ has the dimension of height, and $F=\lambda W$; the proportionality holds only infinitesimally, although it holds at any one point $P(\vec{r}, t)$, then the proportionality factor is a function $\lambda(\vec{r}, t)$, i.e., $F=\lambda(\vec{r}, t) W$. The general infinitesimal law is the "principle of mechanics":

$$
d v^{2}=d \nu \quad d \nu=\lambda(\vec{r}, t) d y
$$

Euler chose units so that $\nu$ was a true height; this was possible by making $n=\frac{1}{2 g}$. But actually, $n=1$; to change to modern units, $\nu$ must be multiplied by $2 g$, so that Euler's "principle of mechanics" becomes:

$$
d v^{2}=d \nu \quad d \nu=2 g \lambda(\vec{r}, t) d y
$$

where $\nu$ does not have the dimension of height (length). For the modern reader, it is better to make in Eq. (6) $n=1$ and $F=\lambda(\vec{r}, t) W$, so that the "principle of mechanics" is stated as:

$$
d v^{2}=2(g \lambda(\vec{r}, t)) d y \equiv 2 \mathcal{G} d y .
$$

The idea of measuring a force by a weight that cancels a motion was not a novelty, when Euler wrote his papers on the dynamic equations. Christiaan Huygens had already used the weight as a measure of the centrifugal force at the onset of motion 11 And so did Galileo in order to find the "impelling force" (Galileo's words) down an incline [15]: the force is equal to a weight that hangs vertically from a pulley at the top of the incline, which keeps the body at rest on the incline.

\section{The meaning of the equation}

As late as 1744 , Jean Le-Rond D'Alembert was criticizing both the concept of force and Newton's axiom II 16,12 accordingly, a relation of the form "cause = effect" is an axiom ([16], p.25) "vague and obscure", and the force in the expression $F=M a$ is not a separate entity, but a name for $M a([16], \mathrm{p} . \mathrm{x})$. But even if the association of a cause with its effect by means of an equation had not been contentious, there remained other problems.

The first problem is the meaning of 'force'. Although Galileo uses a weight to find the force down an incline 17, 18, 13 he so expresses on its nature ([19], p.234):

\footnotetext{
${ }^{11}$ In the De Vi Centrifuga, Huygens contrives the following physical system: a person stands at the top of a rotating vertical wheel, holding a thread with a stone hanging from its bottom. At the beginning of the motion, when the person is at the top of the wheel, the tendency of the stone to move away from the center is balanced by its weight, and goes through the center of the circle. This holds at any point of the circle taken individually, as if at each instant the motion starts anew.

${ }^{12}$ D'Alembert recognizes that the law of inertia requires a "cause" for motion to begin; however $([16]$, p.xi) "... causes can only be known by their effects, and we entirely ignore the nature [of these causes] ...".

${ }^{13}$ Historians have recognized in Galileo's calculation of the force down an incline an attempt to formulate a dynamics. To Max
}

[w] hat I am asking you is not the name of the thing ["gravity"], but its essence [...]. But we do not really understand what principle or what force it is that moves stones downward [...].

In the seventeenth century, the law of inertia did not necessarily imply the existence of an independent entity (the force) in accelerated motions. According to René Descartes, circular motion results, when the tendency to move away from the center of a circle is cancelled by some obstacle ${ }^{14}$ Although Huygens used the weight to measure the force in circular motions, he studied circular motions as an approach to the pendulum [20]; according to H.M. Bos (21], p.607) "[f]orce in the Newtonian sense, could never count as a fundamental principle [...]", and ([21], p.609) "there is no mechanistic philosophy in the Horollogium Oscillatorium [where Huygens first stated the theorems leading to the centrifugal force]" . In Euler's approach, any force has the nature of a weight; then the mass that enters in the expression of the weight is ontologically equivalent to the mass that enters the equation of motion. Nowadays, this is called "equivalence principle".

The second problem is that the choice of a specific equation needs separate justification. The law at most states that the acceleration, $\vec{a}$, is a function of the force, but it does not state what function it is, for example it could be $\vec{F}=m a^{\frac{2}{3}} \hat{a}$. Similar criticism was raised by Daniel Bernoulli; in D. Speiser's account of Daniel Bernoulli's Examen principiorum 22]:

$$
\begin{aligned}
& {[\ldots] \text { a priori nothing seems to prevent a law of the }} \\
& \text { form } d v=p^{2} d t \text { or } d v=p^{3} d t \ldots \text { or even } d v=\sqrt{p} d t \text { or } \\
& d v=f(p) d t \text {, etc. Incidentally, this way of reasoning } \\
& \text { makes c1ear that Bernoulli would never have accepted } \\
& \text { the doctrine according to which } d v=p d t \text { is merely a } \\
& \text { definition, for then it would not matter whether one } \\
& \text { writes } p, p^{2} \text { or } \sqrt{p} \text {, etc. (Ref.35, p.4-5). }
\end{aligned}
$$

Euler seems to be directly addressing to Daniel Bernoulli $(p$ is $F)([5]$, p.56):

Jammer, it is ([17, p.101) "[...] perhaps the first statement of a unified concept of static and dynamic forces". Richard Westfall calls attention to Galileo's conviction that ([18], p.23) "the natural tendency of heavy bodies to fall can provide the standard of measurement for a quantitative dynamics"; he recognizes that the calculation $([18$, p.26) "[...] embodied a dynamic equivalent of the commensurability of impressed force and weight in [Galileo's] De motu".

${ }^{14}$ When a stone rotates in a sling, describing a circle, and leaves the sling, it moves uniformly along a line tangent to the circle (disregarding gravity). Choosing as center of coordinates the center of the circle described by the sling, the uniform velocity along the tangent line has two components, the component perpendicular to the radius vector, and the component along the radius vector; at the point where the stone leaves the circle (the onset of the inertial motion), the radius vector is the radius of the circle. The circular motion of the stone while it is in the sling happens, because the radial component is cancelled by some obstacle (the sling itself), i.e., by cancelling an already existing motion - the tendency to fly away from the center - and need not an external entity. 
152. Therefore it is apparent that not only is this theorem $[d v \propto p d t]$ true, but also it is true by necessity, as thus it would involve a contradiction to put $\left[d v \propto p^{2} d t\right]$ or $\left[d v \propto p^{3} d t\right]$ or another function in place of $[p]$. All of which and equally commendable are considered by the most distinguished Daniel Bernoulli in Comment. Tom.I, and I have been greatly influenced with the rigor of the demonstration of these propositions.

Euler indeed produces Eq. (3) (or Eq. (4)) and Eq. (6). But it is doubtfull that he can rightfully claim that "not only is this theorem true, but also it is true by necessity"; for one thing, taking D'Alembert at his own word, he would certainly not agree with Euler. Other difficulties can be added: there is no guarantee that Eq. (4) and Eq. (6) contain all the elements necessary to describe point mass dynamics; furthermore, there is nothing in the calculations that points to the nature of $F$. However, once Euler makes an analogy with the galilean law, the equations acquire dynamic content.

The crucial point in the "principle of mechanics" is to treat a trajectory as a succession of uniformly accelerated infinitesimal motions. Analytically it means that the differential equations are second order in time 15 geometrically, it means ([23], p.69):

66. For a body to move with a given speed in a circle, it must continuously be driven towards the [center] of the circle $[\ldots]$.

It should be emphasized that it is driven towards the center with an uniformly accelerated motion ${ }^{16}$ Derek Whiteside recognizes the import of second order differentials to the shaping of Isaac Newton's dynamic ideas ([24], p.108):

[...] the continuous growth during the period 1664-84 of Newton's expertise with the various orders of the infinitely small was a significant conditioning factor on the effective expression and forceful pursuance of his dynamical research.

Finally, Euler states a recipe: "it is possible to find from [the equations] the values $x, y, z$, for any elapsed time $t$, consequently the place where the body is".

\section{Supplementary material}

The following online material is available for this article:

Appendix A

Appendix B

${ }^{15}$ If the equation contained the first order derivative, the "instantaneously constant" quantity would be $\vec{v}=\frac{d \vec{r}}{d t}$; if it contained the third order derivative, $\frac{d \vec{a}}{d t}=\frac{d^{3} \vec{r}}{d t^{3}}$, and so on.

${ }^{16}$ The geometric content is best seen in the synthetic approaches of Huygens's and Newton's. If segments on the tangent are of first order in time, then segments between the tangent and the osculating circle, or between the tangent and the orbit are second order in time.

\section{Acknowledgments}

I thank Professor Allan Franklin for his comments on another version, which led me to change the paper in a crucial way; I am grateful for the time he dedicated to it, and honored with his friendship. I thank Professor Ian Bruce for the permission to quote from his translation of Euler's Mechanica, and for helpful comments. I thank Professor Niccolò Guicciardini for the following comment on an early version (private communication): "[b]riefly said: your work is excellent from a technical and philosophical point of view, but it does not provide a reliable and complete (as far as it is possible) narrative of the development that led to Euler's work".

\section{Referências}

[1] L. Euler, Mémoires de l'Académie des Sciences de Berlin 3, 93 (1747), republished in Commentationes Astronomica Leonhardi Euleri Opera Omnia (Societatis Scientiarum Naturalium Helveticæ, Geneva, 1960), serie secunda, v. XXV, edited by M. Schürer, p. 1-44.

[2] L. Euler, Mémoires de l'Académie des Sciences de Berlin 6, 185 (1750), republished in Leonhardi Euleri Opera Omnia, serie secunda, v. V, edited by J.O. Fleckenstein (Societatis Scientiarum Naturalium Helveticæ, Geneva, 1957), p. 81-108.

[3] L. Euler, Mémoires de l'Académie des Sciences de Berlin 4, 1898 (1748), republished in Ref.2, p. 38-63.

[4] L. Euler, Mémoires de l'Académie des Sciences de Berlin 7, 169 (1751), republished in Ref. 2, p. 152.

[5] L. Euler, Mechanica sive motus scientia analytice exposita, 1736. Republished in: Leonhardi Euleri Opera Omnia (Societatis Scientiarum Naturalium Helveticæ, Geneva, 1912), serie secunda, v. I, edited by P. Stackel. Translated by Ian Bruce in "The Euler Archive", Mathematical Association of America, http://eulerarchive. maa.org, acessed on October 17, 2014.

[6] Galileo Galilei, Dialogues Concerning the Two New Sciences, 1638. Translated by Henry Crew and Alfonso de Salvio in The Great Books of the Western World, v. 28 (Encyclopædia Britannica, Chicago, 1952).

[7] M. Clagett, The Science of Mechanics in the Middle Ages (The University of Wisconsin Press, Madison, 1959).

[8] E.J. Dijksterhuis, The Mechanization of the World Picture (Pythagoras to Kepler) (Oxford University Press, Oxford, 1961), translated into English by C. Dikshoorn.

[9] I. Bernard Cohen, The Birth of a New Physics (Heinemann Educational Ltd, Portsmouth, 1961), quoted from the revised edition, Penguin, New York, 1992.

[10] J.L. Lagrange, Mécanique Analytique (Mallet-Bachelier, Paris, 1788), quoted from the 1853 edition, p. 212.

[11] I. Bernard Cohen, in: The Annus Mirabilis of Sir Isaac Newton (1666-1966), edited by R. Palter (The MIT Press, Cambridge, 1970), p. 143-185

[12] C.A. Truesdell, in: Leonhardi Euleri Opera Omnia (Societatis Scientiarum Naturalium Helveticæ, Geneva, 1960), serie secunda, v. X and XI.

[13] P.M. Cardozo Dias, Archive for History of Exact Sciences 54, 67 (1999). 
[14] H. Goldstein, Classical Mechanis (Addison-Wesley, Reading, Massachusetts, 1950).

[15] Galileo Galilei, Les Mechaniques de Galilée, 1626. Translated into French by Father Marin Mersenne, 1634, (reprinted by Presses Universitaires de France, Paris, 1966), p. 55 .

[16] J.R. D'Alembert, Traité de Dynamique, 1744 (Chez Fuchs, Paris, 1796), 2nd ed.

[17] M. Jammer, Concepts of Force (A Study in the Foundations of Dynamics) (Harvard University Press, Cambridge, 1957).

[18] R. Westfall, Force in Newton's Physics (The Science of Dynamics in the Seventeenth Century) (American Elsevier, New York, 1971).

[19] Galileo Galilei, Dialogue Concerning the Two Chief World Systems, 1632. (University of California Press, Los Angeles, 1967), translated and commented by Stillman Drake.

[20] J. Yoder, Unrolling Time (Christiaan Huygens and the Mathematization of Nature) (Cambridge University Press, Cambridge, 1988).

[21] H.M. Bos, in: Dictionary of Scientific Biography (Charles Scribner's Sons, New York, 1970), v. 5 and 6, edited by C.C. Gillispie, quoted from the 1972 print, p. 597-613.

[22] D. Bernoulli, "Examen principiorum mechanicæ [...]", 1726, in "Mechanik", Die Werke von Daniel Bernoulli (Springer, Basel, 1987), Band 3, edited by David Speiser, p. 126-142.

[23] L. Euler, Anleitung zur Naturlehre [...], 1782. Republished in: Leonhardi Euleri Opera Omnia (Societatis Scientiarum Naturalium Helveticæ, Geneva, 1926), series tertia, volumen primum, edited by E. Bernoulli, R. Bernoulli, F. Rudio and A. Speiser, p. 449-560, quoted from the translation by E. Hirsch, The Euler Archive, Mathematical Association of America, http: //eulerarchive.maa.org, acessed on October 17, 2014.

[24] D.T. Whiteside, History of Science 5, 104 (1966). 